\title{
Estimating turbulence in images
}

\author{
Miranda van Iersel and Alexander M.J. van Eijk \\ TNO Defence, Security and Safety, Oude Waalsdorperweg 63, The Hague, The Netherlands
}

\begin{abstract}
Atmospheric turbulence can cause severe blurring and scintillation in images. These distortions result in less detailed images. Using only image enhancement techniques can improve the quality of the images to some extend, but usually not enough to see all details. Turbulence correction techniques, such as post-processing techniques using a deconvolution, are developed and show good results. A more profound understanding of atmospheric turbulence, and especially the impact of turbulence on the image quality, might help to improve the quality of these images even further.

To this end, we present a simple method of estimating the shape and size of the point spread function. The method is tested using images from an ongoing trial in South-Africa.
\end{abstract}

Keywords: Atmospheric turbulence, image correction, filtering, blurring, scintillation, deconvolution

\section{INTRODUCTION}

The performance of imaging systems is limited by the atmosphere between the object and the imaging sensor. The fluctuations of the index of refraction in the atmosphere are the cause of wave front distortions, which in turn are responsible for blurring and scintillation in the images. These effects are responsible for the disappearance of details in an image. The effects of atmospheric turbulence in images can become problematic when one is interested in obtaining highly detailed images.

These effects of blurring and scintillation have been known for quite some time in the field of astronomy and correction techniques are routinely used to enhance the images. There is one major difference between the field of astronomy and ground-to-ground imaging (e.g. imaging systems used for harbour / compound security, etc.). The images used in astronomy are normally taken over a (near-)vertical path through the atmosphere, whereas ground-to-ground imaging invokes a (near-)horizontal path. Consequently, the light for ground-toground imaging over a distance of several kilometers traverses more of the atmospheric boundary layer, a zone extending from the surface to typically two kilometers height and containing relatively strong turbulence. In turn, this causes more severe image distortions than in astronomical imaging.

Over the years different methods have been developed to correct the effects of atmospheric turbulence in images. Roggeman and Welsh ${ }^{1}$ divide these techniques into three categories: 1) adaptive optics techniques, 2) post-processing techniques, and 3) a combination of the two previous classes. The adaptive optics techniques are hardware-based techniques. The phase aberrations of an incoming wave front are measured and mechanical means (like a deformable mirror) are used to correct the effects. The correction is made before an image is recorded. The post-processing techniques make use of software-based methods to correct the images after they are recorded. One advantage of this category of techniques is that, in principle, it can be used on any image sequence, regardless of the imaging system that is used to record the images. There are different post-processing techniques that can be used to correct for the atmospheric turbulence in images. The so-called lucky imaging technique is one possible technique. With this method a large set of short exposure images are taken. From this set of images the best images, taken at a moment of relatively quiet atmosphere, are selected, aligned and added. In this way a new enhanced image is constructed. The idea was first introduced by Fried. ${ }^{2}$ Another possibility to correct for atmospheric turbulence are deconvolution methods.

In this paper we will only consider (post-processing) deconvolution techniques to correct for the atmospheric turbulence in images. In section 2 we present some deconvolution methods that were considered and we show

Corresponding author: M. van Iersel (miranda.vaniersel@tno.nl)

Free-Space Laser Communications X, edited by Arun K. Majumdar, Christopher C. Davis,

Proc. of SPIE Vol. 7814, 78140Q - ( ) 2010 SPIE · CCC code: 0277-786X/10/\$18 · doi: 10.1117/12.861162 
results of these techniques on an image sequence containing modest atmospheric turbulence. The results are best shown in a movie, which is impossible in a paper. In section 3 we shortly explain an on-going experiment taking place in South Africa. In this experiment scintillation, blurring and refraction effects are measured together with meteorological data. The data of this experiment can be used to try and estimate the point spread function in images. Section 4 will give some conclusions and an outlook to future work.

\section{TURBULENCE CORRECTION IN IMAGES}

One option to correct for atmospheric turbulence consists of using deconvolution methods. These methods assume that the recorded image, $i(x, y)$, is a convolution between the point spread function (PSF), $h(x, y)$, and the true image, $t(x, y)$, and some noise, $n(x, y)$ :

$$
i(x, y)=h(x, y) * t(x, y)+n(x, y) .
$$

Here $*$ is the convolution operator. The point spread function (PSF) can be considered as the degree to which the light of a point source is blurred in the spatial domain.

It is well known that in the Fourier domain the convolution operator becomes a normal multiplication and it is often more convenient to take the Fourier transform of the problem. Taking the Fourier transform of Eq. (1) gives

$$
I(u, v)=H(u, v) \cdot T(u, v)+N(u, v),
$$

where $I(u, v), H(u, v), T(u, v)$, and $N(u, v)$ are the Fourier transforms of the recorded image, $i(x, y)$, the point spread function, $h(x, y)$, the true image, $\mathrm{t}(x, y)$, and the noise, $n(x, y)$.

There are different deconvolution methods, which differ in the way the expression for the PSF is found. A blind deconvolution estimates $h(x, y)$ from the image, usually in an iterative process. When the blurring in an image is due to a PSF that is spatially-invariant and exactly known, the Wiener filtering is an appropriate deconvolution process. This method provides an estimation for the image on the basis of the least mean square error between the estimated image and the true image, which can be expressed as

$$
T(u, v)=\left[\frac{\left|H(u, v)^{2}\right|}{H(u, v) \cdot\left(\left|H(u, v)^{2}\right|+\frac{S_{n}(u, v)}{S_{o}(u, v)}\right)}\right] \cdot I(u, v) .
$$

Here $S_{n}(u, v)$ and $S_{o}(u, v)$ are the power spectral densities of the noise and of the image.

Similarly one can take the deconvolution with a sharpening filter resulting in

$$
T(u, v)=F^{n}(u, v) \cdot I(u, v),
$$

where $F^{n}(u, v)$ is the $n$-th order derivative of some function. This can be a simple sharpening filter, a Gaussian $n$-th order derivative, etc.

Other deconvolution methods, like the Lucy-Richardson deconvolution, are described in Seiffer, ${ }^{3}$ who also show some results of the different methods. Several deconvolution methods are also compared in Lemaitre. ${ }^{4}$ In the next section we will present some results of different methods used to compensate for atmospheric turbulence in an image. We have used the convolution methods described above, i.e., the blind deconvolution, the Wiener filter and unsharp masking filters. The results of these methods are presented below.

After the deconvolution was performed, we used some standard image processing techniques to try to get images where the details were even more visible. The standard image enhancement suite from $\mathrm{TNO}^{5}$ was used, which contains, among others, dynamic super-resolution (DSR), local adaptive contrast enhancement (LACE), image stabilization, and moving object detection (MOD).

Small and fast moving objects are important in military applications and it is important that they are preserved in an image sequence. However, it requires some extra effort to contain these small and fast moving objects in an image sequence when applying enhancement algorithms to these images. Using a simple time 
integration, these objects will be smeared out and become almost invisible. Whereas the displacement of large, slow moving objects (like a boat) between two consecutive images in a sequence is small (in pixels) in comparison to the size of the object, this is not the case for small and fast moving objects. To contain these objects in an image sequence, it is possible to use a moving object detection (MOD) algorithm. Such an algorithm detects the objects, which then can be separated from the rest of the image. Enhancement algorithms can be applied to the image and the moving objects can be placed back in afterwards. Selecting the size of the objects to be detected by the MOD algorithm, one has to keep in mind that atmospheric turbulence causes small spatial and temporal variations in images as well. This means that there is a limit to the minimum size of an object to be detected by such an algorithm.

\subsection{Results of turbulence correction}

An image sequence, of a ship at a distance of $12.5 \mathrm{~km}$, was recorded at the tower of TNO (at a height of approx. $45 \mathrm{~m}$ ) in The Hague, the Netherlands. A snapshot image of this sequence is shown in figure 1a. Since strong winds were present at the time of recording, the camera platform on top of the TNO tower was not a stable platform. As a result, the position of the ship in the image moved considerably and it was difficult to estimate effects of atmospheric turbulence on the image quality. Image stabilization was performed on the original image to get a sequence of images in which the atmospheric turbulence was more visible. Several deconvolution methods were tested using the stabilized (original) image sequence. A deconvolution with an inverse Wiener filter, a blind deconvolution, and a deconvolution with a sharpening filter were all tested. The sizes of the width, $\sigma$, of the PSF were determined by trial and error. The shape of the PSF was taken to be Gaussian. Results of these different methods are shown in figure 1. The deconvolution with an inverse Wiener filter (figure 1b) and the blind deconvolution (figure 1c) show some more detail in the images. The image after applying only a deconvolution with a sharpening filter (figure 1d) shows more detail, but this method introduces more noise as well. After applying these methods to the original image sequence, we used the image enhancement suite of TNO to see if the results for each deconvolution could be improved some more. The best results were found in the combination of a deconvolution with a sharpening filter and the image enhancement suite (figure 1e).

An image sequence showing a church, some apartment buildings and birds was used to test the atmospheric turbulence correction methods on images containing small and fast moving objects. A moving object detection (MOD) algorithm was used to detect the birds. These moving objects were removed from the images, the images were corrected and afterwards the moving objects were placed back into the scene. Figure 2 shows a snapshot image of the original sequence (a), an image after a deconvolution with a sharpening filter and time averaging (b), and an image after using MOD, a deconvolution with a sharpening filter, and a running average (c). Looking at these images, it is clear that the bird, present in the original image, is smeared out after a simple time integration is used on the whole image (see red circle in figure 2). The information of the moving object is lost in this case, which makes it impossible to detect it. Using the MOD algorithm, the bird is preserved and clearly some in the image.

Unfortunately there were no meteorological parameters recorded at the time of recording of the previously used image sequences. Looking at the image sequences one can recognize that atmospheric turbulence is present in the images and that it is not very strong. (This is much more clearly shown in a movie.) The objects in the images are not completely deformed by the turbulence. The shape of the PSF was taken to be a Gaussian and the size was determined by trial and error. It would be interesting to test if our algorithms work for more severe conditions as well. Besides that, it would also be interesting to see if meteorological data can be used to estimate the shape and size of the PSF. For this purpose we use images and meteorological data recorded during the FATMOSE trial in False Bay, South Africa (see section 3).

\section{FATMOSE TRIAL IN FALSE BAY, SOUTH AFRICA}

The FATMOSE (False Bay ATMOSpheric Experiment) trial is a jointly organized trial by TNO and the Institute of Marine Technology (IMT), taking place in False Bay, South Africa. In this section a short description of several elements of the experiment (those that are relevant to the work described in this paper) is given. A more thorough description of the whole trial can be found in de Jong et.al. ${ }^{6}$

Proc. of SPIE Vol. 781478140 Q-3 


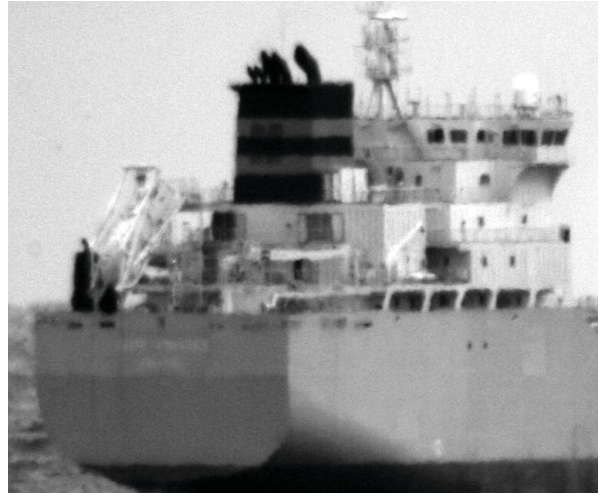

(a)

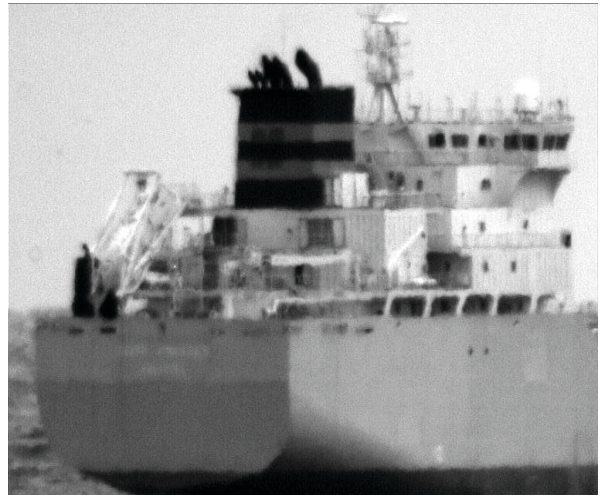

(c)

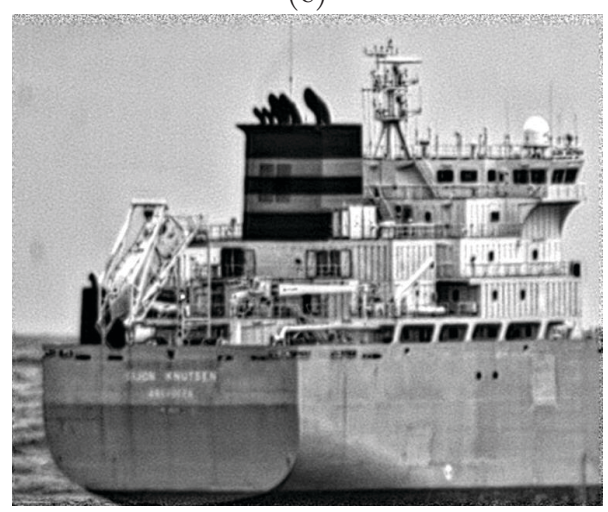

(e)

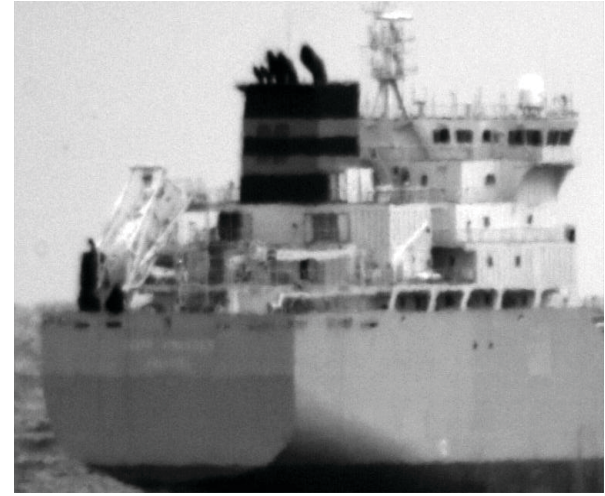

(b)

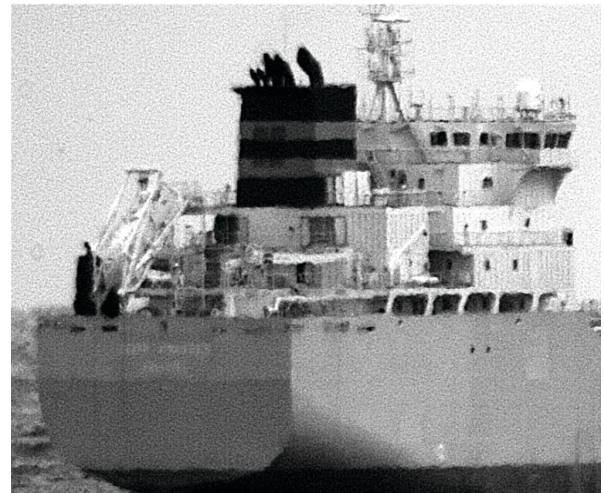

(d)

Figure 1. Snapshot image of the same sequence; original image and images corrected with different algorithms. (a) Original image, (b) Image after applying a deconvolution with a Wiener filter, (c) Image after applying a blind deconvolution, (d) Image after applying a deconvolution with a sharpening filter, (e) Image after applying a sharpening filter and the TNO image processing suite. 


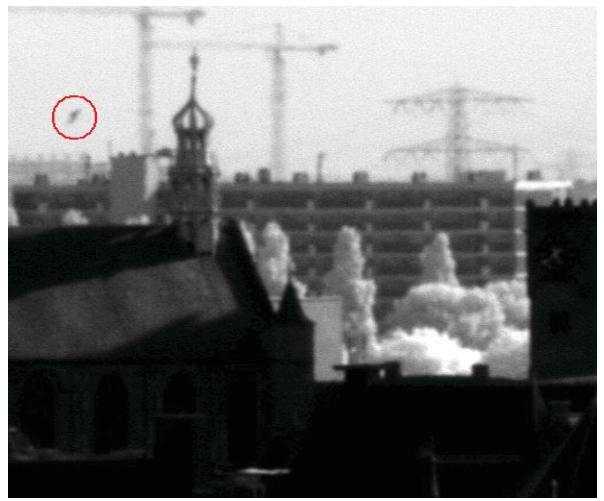

(a)

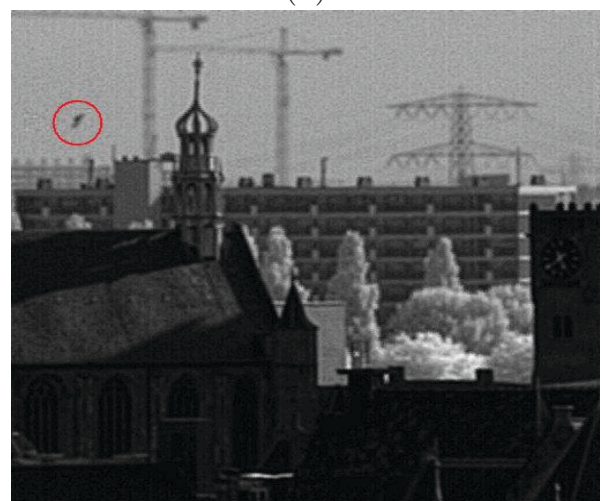

(c)

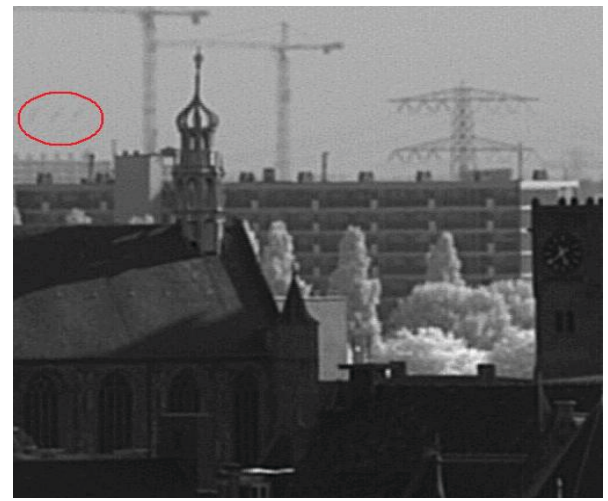

(b)

Figure 2. Image of the same scene containing small and fast moving objects (birds). (a) Original image, (b) Image after applying a deconvolution with a sharpening filter and time averaging, (c) as (b) but with moving object detection.

During this trial scintillation, blurring, and refraction effects are measured over the better part of the year, allowing us to capture seasonal variations in the area. The mean measurement path is in the horizontal direction, $15.7 \mathrm{~km}$ long and completely over sea (see figure 3). Several instruments are used in this trial, among them a BLS900 scintillometer and a Marlin F-033B camera. The receivers of the instruments are installed in Simon's Town at a height of $14.5 \mathrm{~m}$ above mean sea level. The camera is looking at an array of lights mounted at a beach station at Strandfontein. The heights of the upper and lower lamps are at 9.7 and 5.8 m above mean sea level. The height of the two other lamps is $8.7 \mathrm{~m}$ above mean sea level and these lamps have a horizontal separation of $10 \mathrm{~m}$ (see figure 4). The sources for the BLS900 are placed on the Roman Rock Lighthouse, about $1.8 \mathrm{~km}$ off the coast near Simon's Town, at a height of approximately $15 \mathrm{~m}$ above mean sea level. Standard meteorological parameters are recorded by a weather station at Roman Rock. Additional turbulence data is recorded by a sonic anemometer, also mounted on Roman Rock.

\subsection{Data from the FATMOSE trial}

The data recorded during the FATMOSE trial show variations which are in line with the seasonal variations. The air-sea temperature difference (ASTD) is distributed around zero degrees, with variations to negative ASTD of approximately minus three degrees and positive ASTD of seven degrees (or occasionally more). From this distribution we see that during the trial period days with stable conditions appeared as well as days with unstable conditions. The wind speed was measured with a weather station placed on Roman Rock. Wind speeds range from 0 to $15 \mathrm{~m} / \mathrm{s}$, with occasional wind speeds of $20 \mathrm{~m} / \mathrm{s}$ of more. The refractive index structure parameter, $C_{n}^{2}$, was measured with a BLS900 scintillometer. The values of $C_{n}^{2}$ range from $10^{-14} m^{-2 / 3}$ to almost $10^{-17} m^{-2 / 3}$.

A few example images, taking with the Marlin F-033B camera, are shown in figure 5. These images are recorded when different strengths of the atmospheric turbulence occurred; $C_{n}^{2}=1.3 \times 10^{-15} \mathrm{~m}^{-2 / 3}$ for December 


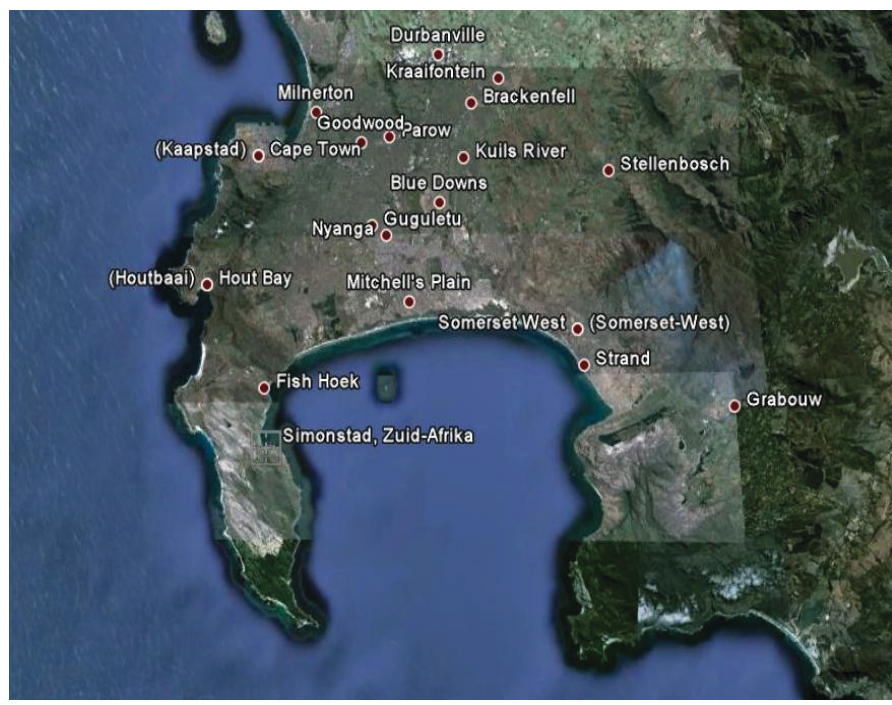

(a)

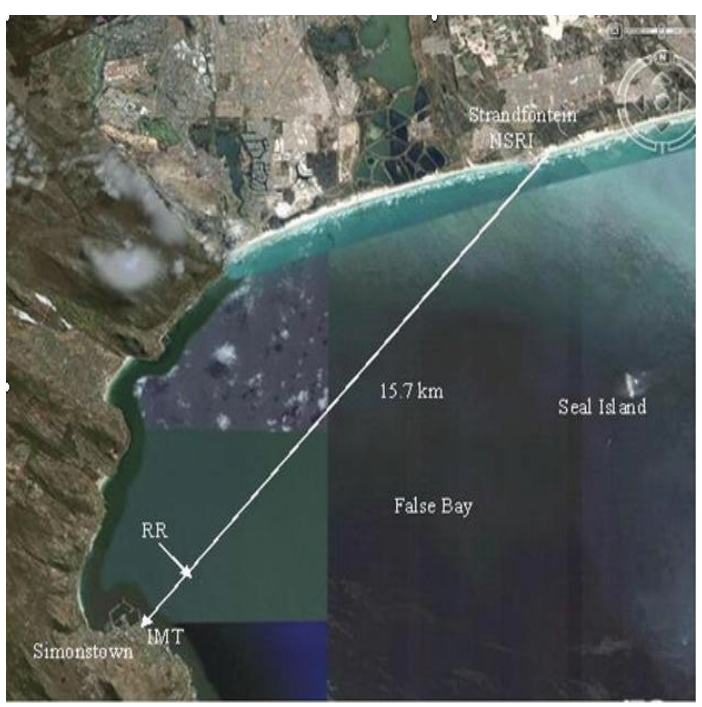

(b)

Figure 3. (a) Measurement path over False Bay, South Africa. (b) Instruments are placed at Simon's Town, sources are placed at Strandfontein and at Roman Rock (RR), where a weather station is situated as well.

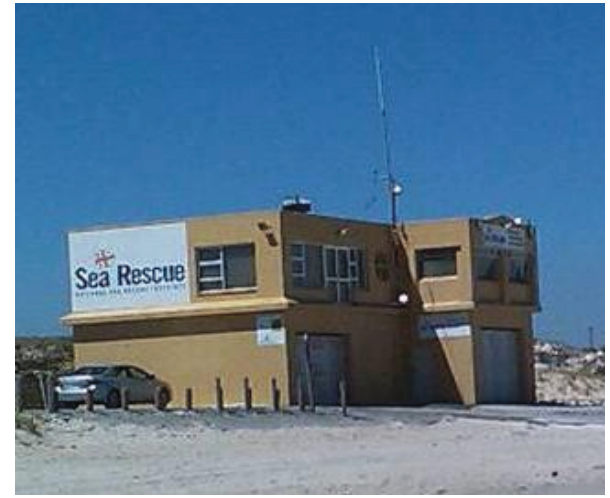

(a)

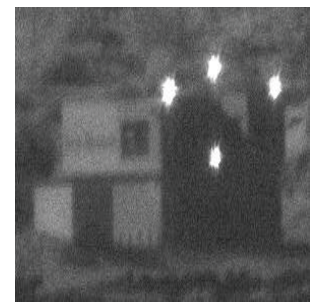

(b)

Figure 4. An array of lights mounted at the beach station at Strandfontein. (a) Visual image, (b) Infrared image. 


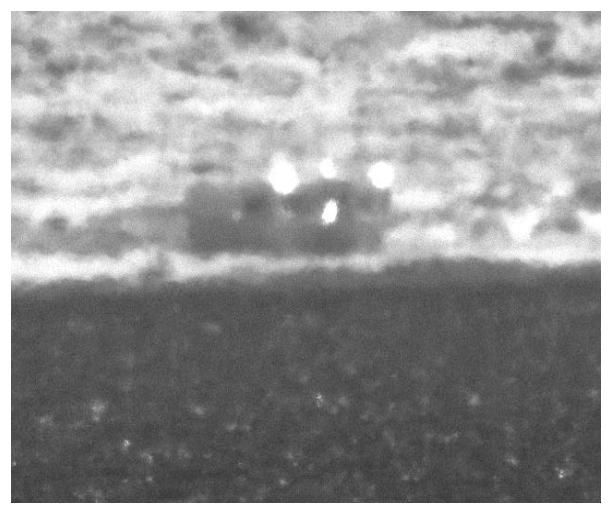

(a)

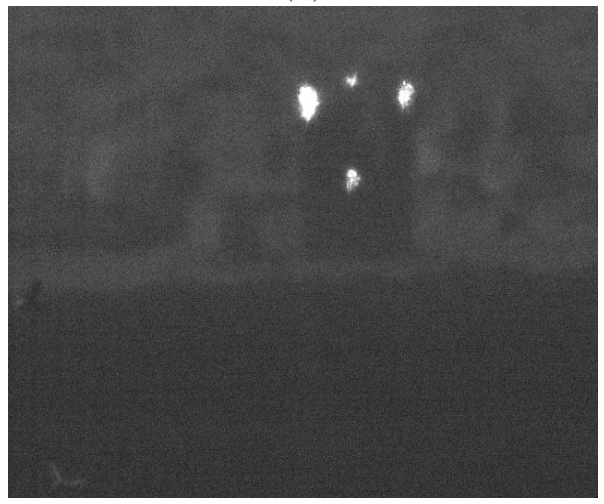

(c)

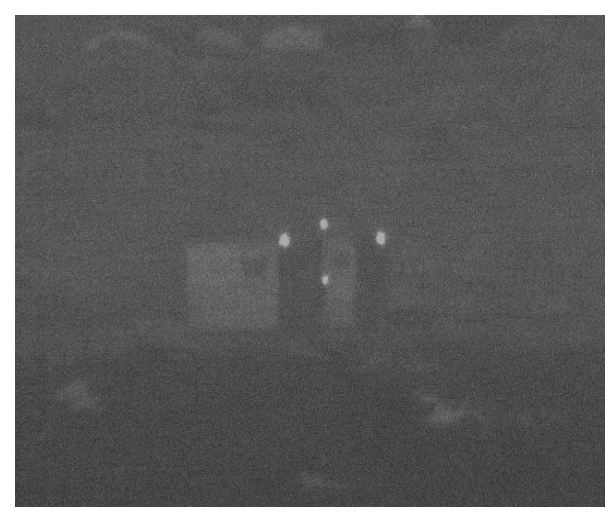

(b)

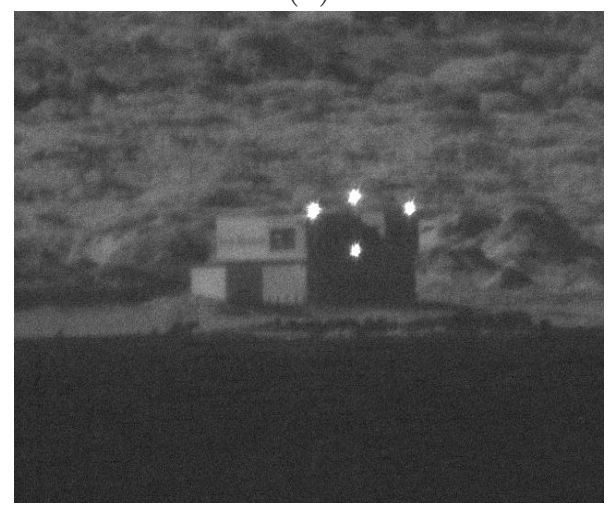

(d)

Figure 5. Images of the sources at Strandfontein taken at different days. (a) December 8, 2009 (b) December 13, 2009 (c) April 12, 2010 (d) April 20, 2010.

8, 2009, $C_{n}^{2}=2.3 \times 10^{-16} m^{-2 / 3}$ for December 13, 2009, $C_{n}^{2}=1.0 \times 10^{-14} m^{-2 / 3}$ for April 12,2010, and $C_{n}^{2}=1.9 \times 10^{-16} m^{-2 / 3}$ for April 20, 2010. Both December 13, 2009 and April 20, 2010 show low atmospheric turbulence. The wind speeds occurring during these days are different. On December 13, 2009 the wind speed was over $15 \mathrm{~m} / \mathrm{s}$ during the whole day, while on April 20, 2010 the wind speed was below $5 \mathrm{~m} / \mathrm{s}$ during the whole day. In table 1 the corresponding meteorological conditions are given for the days presented in figure 5 .

\begin{tabular}{|c|c|c|c|c|c|c|c|c|}
\hline Date & Time & $C_{n}^{2}\left[\mathrm{~m}^{-2 / 3}\right]$ & $T_{\text {air }}\left[{ }^{\circ} \mathrm{C}\right]$ & $A S T D\left[{ }^{\circ} \mathrm{C}\right]$ & $P[\mathrm{mB}]$ & $R H[\%]$ & $V_{\text {wind }}[\mathrm{m} / \mathrm{s}]$ & Winddir $[$ deg $]$ \\
\hline $08-12-2009$ & 10.15 & $1.3 \times 10^{-15}$ & 20.28 & 1.96 & 1011.1 & 69.0 & 7.9 & 342.3 \\
\hline $13-12-2009$ & 19.00 & $2.3 \times 10^{-16}$ & 18.24 & 0.65 & 1016.9 & 70.5 & 18.9 & 153.8 \\
\hline $12-04-2010$ & 14.45 & $1.0 \times 10^{-14}$ & 22.17 & 2.51 & 1012.8 & 66.2 & 2.4 & 150.1 \\
\hline $20-04-2010$ & 15.35 & $1.9 \times 10^{-16}$ & 18.23 & 0.02 & 1021.0 & 58.7 & 4.2 & 281.6 \\
\hline
\end{tabular}

Table 1. Meteorological parameters corresponding to the images in figure 5.

\subsection{Estimating the point spread function}

In the methods described in section 2 the shape of the PSF was taken to be a Gaussian and the size of the PSF was determined by trial and error. It would be better if one could estimate the size and shape of the PSF from the image or using additional measurements. Yitzhaky et.al. ${ }^{7}$ describe a technique to estimate the turbulence and aerosol modulation transfer functions (MTF) using meteorological parameters. The MTF for long exposure (LE) and short exposure(SE) are given by:

$$
M T F_{L E}=\exp \left(-57.3 \nu^{5 / 3} C_{n}^{2} \lambda^{-1 / 3} R\right)
$$




$$
M T F_{S E}=\exp \left(-57.3 \nu^{5 / 3} C_{n}^{2} \lambda^{-1 / 3} R\left[1-\mu\left(\frac{\lambda \nu}{D}\right)^{1 / 3}\right]\right)
$$

Here $\nu$ is the angular spatial frequency, $\lambda$ the radiation wavelength, $R$ the path length, $D$ the aperture diameter, and $\mu$ is a coefficient taken to be 0.5 in the far field and 1 in the near field. The value for the refractive index structure parameter, $C_{n}^{2}$, is estimated by a model using standard meteorological parameters and is given by Yitzhaky et.al. ${ }^{7}$ by

$$
\begin{aligned}
C_{n}^{2} & =3.8 \times 10^{-14} W+2 \times 10^{-15} T-2.8 \times 10^{-15} R H+2.9 \times 10^{-17} R H^{2}-1.1 \times 10^{-19} R H^{3} \\
& -2.5 \times 10^{-15} W S+1.2 \times 10^{-15} W S^{2}-8.5 \times 10^{-17} W S^{3}-5.3 \times 10^{-13},
\end{aligned}
$$

where $W$ is a temporal-hour weight, $T$ is the air temperature in $K, R H$ is the relative humidity in \%, and $W S$ is the wind speed in $\mathrm{m} / \mathrm{s}$.

A more theoretical approach to predict the PSF is given by Potvin et.al., ${ }^{8}$ who derive the PSF from the assumption that it is a Gaussian and that it depends on only six parameters: the scintillation, the displacement vector, the attitude and horizontal and vertical spreads. These six parameters are assumed to be random fields, which only depend on the coordinates of the object and the time.

These theoretical approaches can provide reasonable estimates for the PSF and it is certainly interesting to apply them to the data of the FATMOSE trial. It would be interesting to compare these theoretical estimates to an experimental in-situ measurement of the PSF. The FATMOSE experiment provides such measurements, since the lights shown in figure 4 may be regarded as point sources, which can be used to estimate the size of the PSF directly from the images. And it is this approach that we will follow in the next section.

\subsection{PSF estimates on images of the FATMOSE trial}

To determine the size of the width, $\sigma$, of the PSF we have determined the size of the upper and lower spot in the images recorded during the FATMOSE trial. These value were used in our turbulence correction algorithm. For the image sequence taken on April 20,2010 at 15.35 a spot size of 2.41 was found for the upper spot and a size of 2.43 was found for the lower spot. The value of 2.4 was used as the size of the width, $\sigma$, of the PSF. Figure $6 \mathrm{~b}$ shows an image of the sequence after our turbulence correction algorithms were used. As comparison an image from the original image is shown in figure $6 \mathrm{a}$.

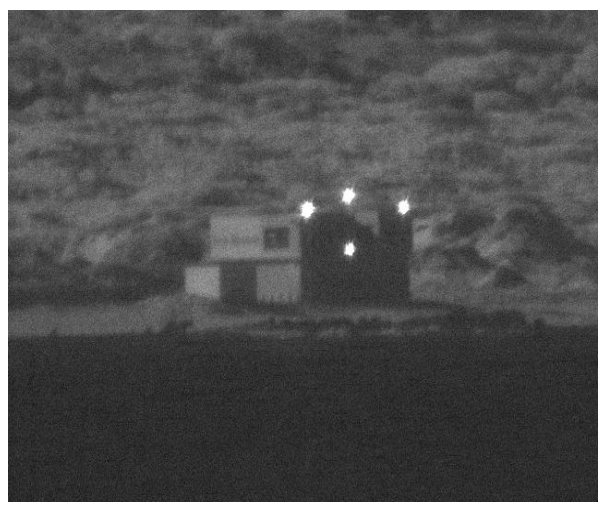

(a)

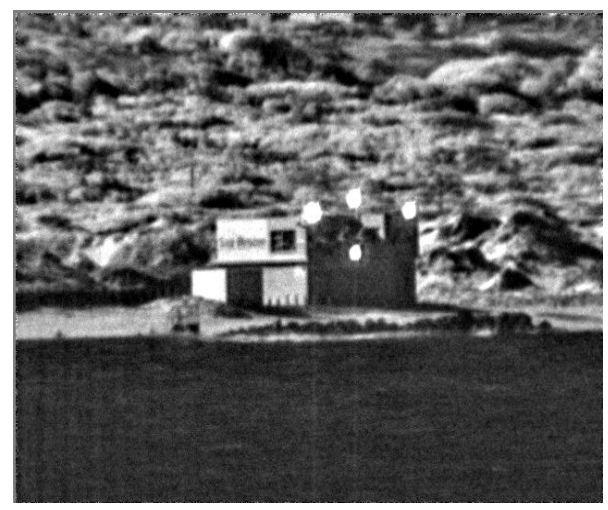

(b)

Figure 6. Image of the sources at Strandfontein taken at April 20, 2010 at 15.35 corrected with our turbulence algorithms.

The result of our algorithms with a PSF width of 2.4 was compared to the results found for different values of this width $\sigma$. In figure 7 the results of our algorithms are plotted for different values of the size of the PSF $(\sigma=0.5, \sigma=1, \sigma=2$, and $\sigma=4)$. Looking at figure 7 we see that the result with a size $\sigma=2$ gives the best result. This value agrees reasonably well with the value of 2.4 measured directly from the images. 


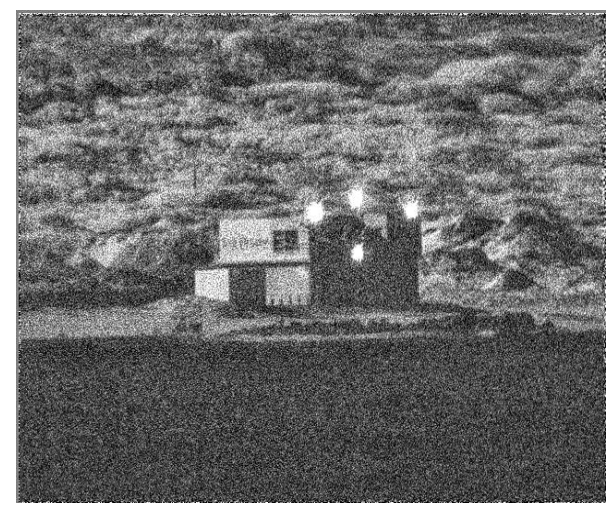

(a)

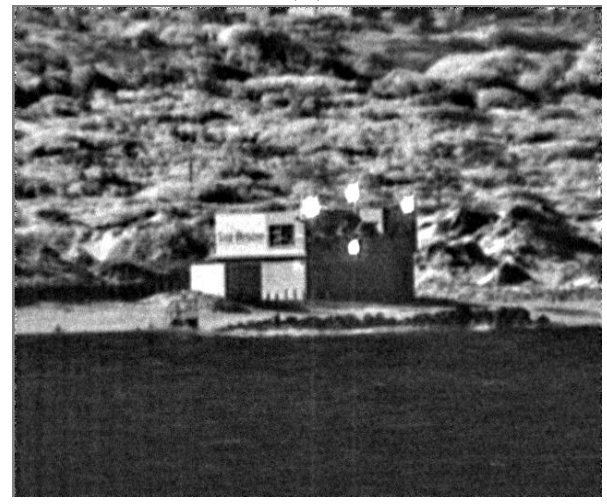

(c)

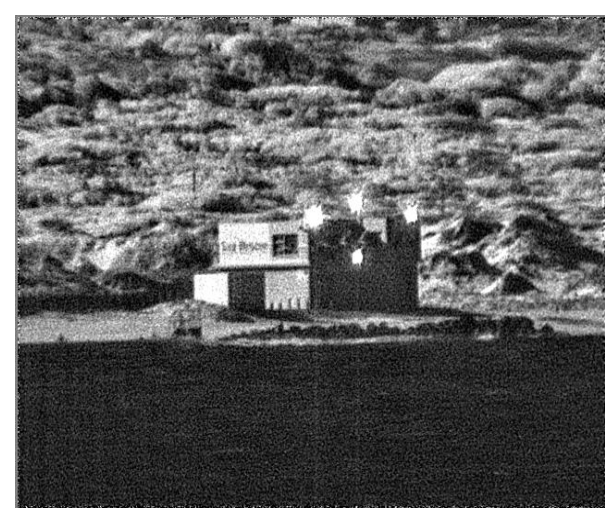

(b)

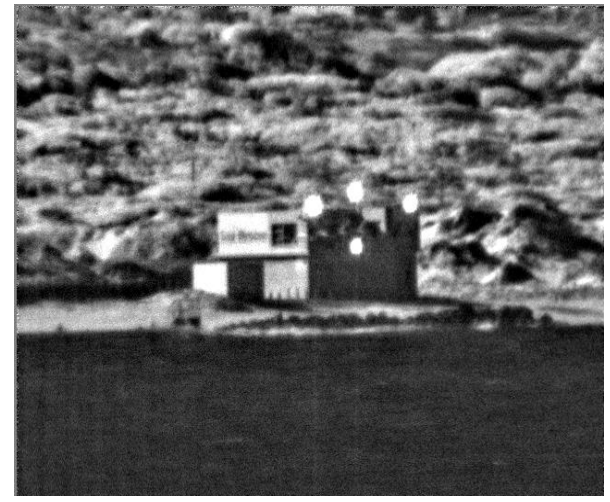

(d)

Figure 7. Images of the sources at Strandfontein taken at April 20, 2010 corrected with different size of the PSF. (a) $\sigma=0.5$ (b) $\sigma=1.0$ (c) $\sigma=2.0$ (d) $\sigma=4.0$.

For other days the measured spot sizes differ somewhat more, e.g. the size measured in the images taken on December 13, 2009 are 1.86 for the upper spot and 1.78 for the lower spot. A PSF width of $\sigma=1.8$ was chosen for our algorithms. Comparing this result to the results found by varying the PSF width we found that the best comparison was found for a size 1.5 and 2.0 (images are not shown). It is in this range that the measured spot size lies.

The images taken during the FATMOSE trial all show the sources, which we have assumed to be a point source. Unfortunately such a point source is not always present in an image sequence. Determining the spot size becomes impossible in that case. Therefore, it would be interesting to apply the methods of Yitzhaky et.al. ${ }^{7}$ and Potvin et.al. ${ }^{8}$ to the images taken during the FATMOSE trial and see if the estimated PSFs coincide with the PSF width measured in the images.

\section{CONCLUSIONS}

Deconvolution methods can be used to compensate for atmospheric turbulence in images. Different deconvolution methods were tested, using different sizes of the width of the point spread function. To test if the quality of the images could be enhanced even further, the standard image enhancement suite of TNO was used after wards. The best result was find in the combination of a deconvolution with a sharpening filter and the image enhancement suite. The shape of the PSF was taken to be a Gaussian and the size of the width of the PSF was determined by trial and error.

Images recorded during the FATMOSE trial in South Africa are used to estimate the size of the width of the point spread function. The spot sizes of the sources in the images where determined and used to estimate the 
value of the size of the PSF. This estimated value was compared to results found with other values and it seems to give the best result in corrected images.

Not all images contain objects which can be assumed a point source. Which means that an estimation of the size of the PSF directly from the image cannot be done for these images. A method to predict the size (and shape) of the PSF is more convenient in that case. The methods to estimate the PSF as proposed by Yitzhaky et.al. ${ }^{7}$ and Potvin et.al. ${ }^{8}$ will be compared to the results presented in this paper.

\section{ACKNOWLEDGMENTS}

Part of the work presented in this paper was sponsored by the Royal Netherlands Armed Forces. The authors would like to thank Arie de Jong, Peter Fritz, and Koen Benoist for their work in the FATMOSE trial.

\section{REFERENCES}

[1] Roggeman, M.C., and B. Welsh, "Imaging Through Turbulence", CRC Press, Boca Raton, USA, 1996.

[2] Fried, D.L., "Probability of getting a lucky short-exposure image through turbulence", Optical Society of America Journal 68, 1651-1658, 1978.

[3] Seiffer, D., "TA 108.019 Laser Beam Propagation and Imaging through severe Environments - Final Report WP05 - High-resolution passive and active imaging", Fraunhofer-IOSB 2010/09, 2010.

[4] Lemaitre, M., J. Blanc-Talon, F. Mériaudeau, and O. Laligant, "Evaluation of Infrared Image Restoration Techniques", SPIE Proc. Vol.6395, 63950R, 2006.

[5] Schutte, K., D.J.J. de Lange, and S.P. van den Broek, "Signal conditioning algorithms for enhanced tactical sensor imagery", SPIE Proc. Vol. 5076, 92-100, 2003.

[6] Jong, A.N. de, P.J. Fritz, K.W. Benoist, A.M.J. van Eijk, and P.B.W. Schwering, "Preliminary results of the FATMOSE atmospheric propagation trials in the False Bay, South Africa, from November 2009 - July 2010, to be published at SPIE Proc.

[7] Yitzhaky, Y., I. Dror, and N.S. Kopeika, "Restoration of atmospherically blurred images according to weather-predicted atmospheric modulation transfer functions", Opt. Eng. 36(11), 3064-3072, 1997.

[8] Potvin, G., J.L. Forand, and D. Dion, "A parametric model for simulating turbulence effects on imaging systems", DRDC Valcartier TR 2006-787, 2007. 